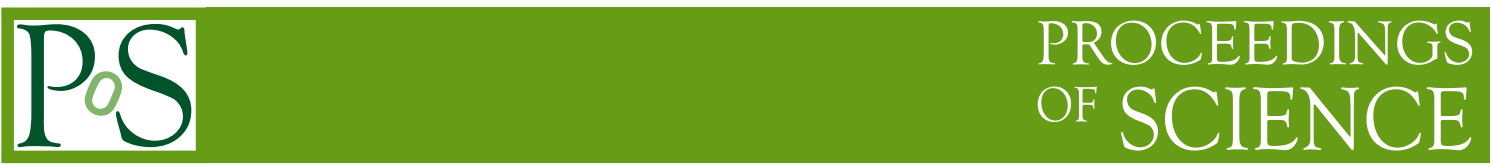

\title{
Searches for sterile neutrinos with IceCube
}

\section{Andrii Terliuk* for the IceCube collaboration ${ }^{\dagger}$}

Deutsches Elektronen-Synchrotron, Platanenallee 6, 15738 Zeuthen, Germany

E-mail: andrii.terliuk@desy.de

Sterile neutrinos are a hypothetical additional neutrino species that do not interact via weak interactions. They can resolve anomalies observed in the data of short baseline, reactor and radiochemical neutrino experiments. The presence of sterile neutrinos, when mixed with the active neutrino states, can lead to deviations in the atmospheric neutrino flux. The IceCube Neutrino Observatory, located at the geographic South Pole, is the first cubic kilometer Cherenkov neutrino detector. It can measure atmospheric neutrinos with a neutrino detection energy down to $10 \mathrm{GeV}$ and up to energies of about $100 \mathrm{TeV}$. We present results from two searches for sterile neutrino mixing using data from IceCube.

XVII International Workshop on Neutrino Telescopes

13-17 March 2017

Venezia, Italy

\footnotetext{
${ }^{*}$ Speaker.

${ }^{\dagger}$ http://icecube.wisc.edu/
} 


\section{Introduction}

Neutrino oscillation is a phenomenon in which neutrinos can be detected in a different flavor eigenstate than initially produced. It is caused by the difference between weak and mass neutrino eigenstates. The effect has been confirmed by a variety of measurements using neutrinos produced in the Sun, nuclear reactors, accelerators and the atmosphere [1]. The data of neutrino oscillation experiments can be interpreted in the framework of three weakly interacting neutrinos, each being a superposition of the three mass (propagation) states.

However, not all experimental data is consistent with the standard three neutrino picture. Anomalies are observed in the data from the LSND [2] and MiniBooNE [3] experiments, as well as at reactors [4] and radiochemical neutrino experiments [5]. The tension between the data of different experiments can be partially resolved by adding new families of neutrinos with a mass difference $\Delta m^{2} \sim 1 \mathrm{eV}^{2}$. However, these new states cannot interact via standard weak interactions due to constraints from the Z-boson decay width measured at LEP [6] and, therefore, are called sterile.

The simplest sterile neutrino model is a " $3+1$ " model, where only one heavier sterile neutrino is added. The mixing between the active states and sterile neutrinos results in modifications of the observed atmospheric neutrino flux and can be probed with data from the IceCube detector. Two strategies to search for sterile neutrino mixing, performed at different energy ranges, are presented in this work. The first strategy uses events with energies above $400 \mathrm{GeV}$ and searches for a resonant enhancement of the sterile neutrino mixing for muon antineutrinos crossing the Earth's core. The second search uses lower energy data from DeepCore, a denser part of IceCube, in the energy range between 6 and $56 \mathrm{GeV}$ to search for deviations from the standard atmospheric neutrino oscillations due to the sterile neutrino mixing.

\section{IceCube and DeepCore}

The IceCube detector [7] uses the antarctic ice as an optical medium to detect Cherenkov light emitted by products of neutrino interactions. The detector instruments about a cubic kilometer of ice between 1450 and 2450 meters below the ice surface. IceCube consists of 5160 Digital Optical Modules (DOMs) grouped in 86 vertical strings with 60 modules each. Seventy-eight of the strings are arranged in a hexagonal grid with a typical lateral separation of $125 \mathrm{~m}$ as shown in the left part of Figure 1. The typical vertical spacing between DOMs is $17 \mathrm{~m}$. The geometry of the detector, DOM efficiency and optical properties of the ice result in a neutrino detection energy threshold of about $100 \mathrm{GeV}$.

DeepCore [8], a denser sub-detector, is located in the central bottom part of IceCube as shown in Figure 1. The antarctic ice has the best optical properties in this region of the detector. DeepCore consists of 8 strings with an average lateral spacing of $50 \mathrm{~m}$. Seven surrounding IceCube strings are also included in the DeepCore fiducial volume. The DOMs on a DeepCore string have a $7 \mathrm{~m}$ vertical separation in the fiducial volume and are instrumented with 35\% higher quantum efficiency PMTs that the conventional IceCube DOMs. The reduced spacing, better efficiency and optical properties of the deep ice lower the energy threshold to about $10 \mathrm{GeV}$. At the IceCube trigger level the rate of neutrino-induced events is about $10^{5}$ lower than the background from muons produced by the 

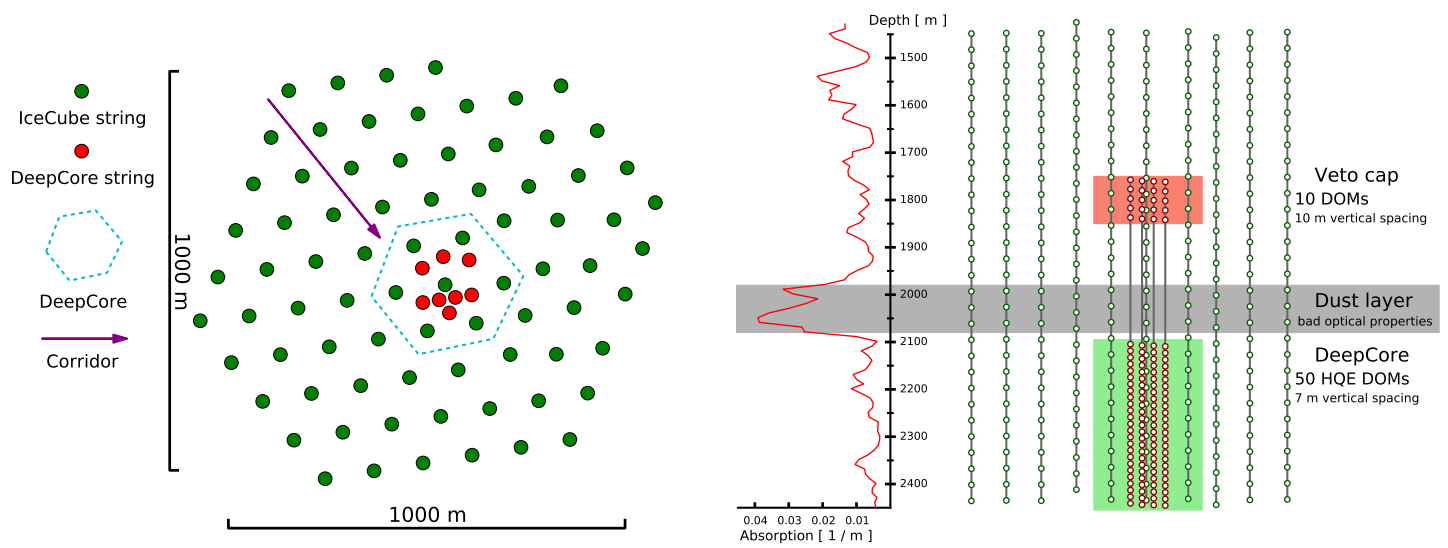

Figure 1: (Left) A top view of IceCube detector. Conventional IceCube strings are marked green and DeepCore strings, which use a denser vertical spacing and high quantum efficiency DOMs, are marked in red. The dashed line encompasses the DeepCore fiducial volume, while veto volume is located outside it. The purple arrow shows an example of a detector corridor, where atmospheric muons can penetrate to DeepCore leaving only few photon hits in the veto region. (Right) A side view of IceCube detector. The standard IceCube DOMs and high quantum efficiency DeepCore DOMs are depicted by green and red circles respectively. The DeepCore fiducial volume is shown in the green shaded region. The light red region is used to improve the veto against down-going atmospheric muons. The red line on the left axis shows the optical photon absorption length as function of depth. The gray area shows the location of the dust layer, a region with short optical absorption lengths.

cosmic ray interactions in the atmosphere. Therefore, outer IceCube strings are used an active veto to reject such background.

\section{Sterile neutrino mixing}

The simplest extension of the standard three neutrino model is the " $3+1$ " model, with one additional sterile neutrino state that is heavier than the three standard mass states. The mixing between the flavor states $\left|v_{\alpha}\right\rangle$, which describe weak interactions, and the mass states $\left|v_{k}\right\rangle$ is

$$
\left|v_{\alpha}\right\rangle=\sum U_{\alpha k}^{*}\left|v_{k}\right\rangle
$$

where $U_{\alpha k}$ are the elements of a unitary mixing matrix. In the standard three neutrino case the mixing matrix can be parametrized in the form of the $3 \times 3$ Pontecorvo-Maki-Nakagawa-Sakata (PMNS) matrix with three mixing angles $\left(\theta_{12}, \theta_{13}\right.$ and $\left.\theta_{23}\right)$ and one CP-violating phase $\delta[9,10]$. An additional sterile neutrino results in three new mixing angles $\left(\theta_{14}, \theta_{24}\right.$ and $\left.\theta_{34}\right)$ and two additional CP-violating phases $\left(\delta_{14}, \delta_{34}\right)$ [11]. IceCube cannot distinguish neutrinos from antineutrinos and has minimal sensitivity to CP-violating phases and, therefore, they are assumed to be zero in this work. The angle $\theta_{14}$ affects mainly electron neutrinos, which have a minor impact on the presented studies, and the value of $\theta_{14}$ is set to zero. These assumptions simplify the sterile neutrino 

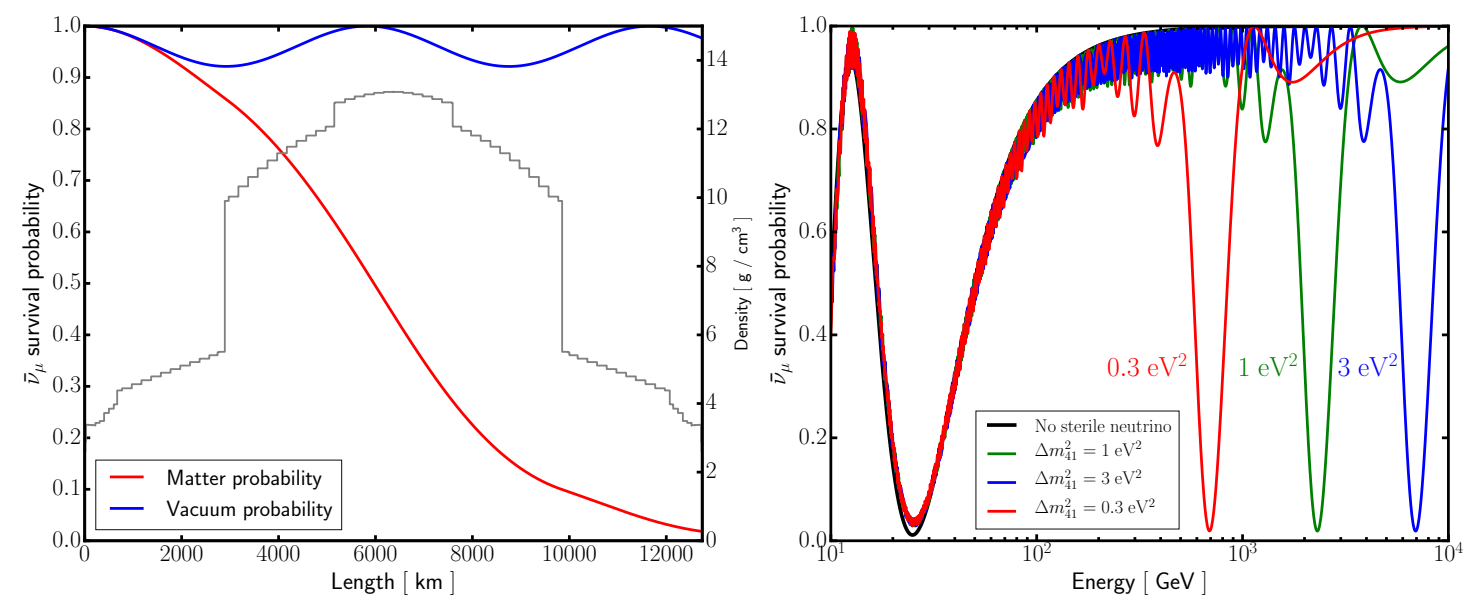

Figure 2: (Left) The survival probability for muon antineutrinos with energy $2.35 \mathrm{TeV}$ crossing the Earth on a diametral trajectory $\left(\cos \theta_{z}=-1\right)$. The values for the sterile neutrino mixing are $\Delta m_{41}^{2}=1 \mathrm{eV}^{2}$ and $\sin ^{2} \theta_{24}=0.02$. The vacuum probability is shown in blue. (Right) The survival probability for neutrinos crossing the Earth's core ( $\left.\cos \theta_{z}=-1\right)$ for different values of the sterile neutrino mass splitting. The sterile neutrino mixing angle is $\sin ^{2} \theta_{24}=0.02$. The black line indicates the case of zero sterile neutrino mixing, i.e. standard neutrino oscillation.

mixing elements [12] of the matrix $U$ as

$$
\begin{aligned}
\left|U_{e 4}\right|^{2} & =0, \\
\left|U_{\mu 4}\right|^{2} & =\sin ^{2} \theta_{24}, \\
\left|U_{\tau 4}\right|^{2} & =\cos ^{2} \theta_{24} \cdot \sin ^{2} \theta_{34} .
\end{aligned}
$$

The presence of matter modifies the neutrino oscillations, which can be observed as an effective change of the mixing parameters. Sterile neutrinos do not undergo weak interactions, which leads to a zero matter potential as they traverse the Earth. On the other hand, all active neutrinos can interact via neutral current (NC) interactions and experience an effective matter potential. The difference between the potentials for the sterile and active neutrinos leads to differences in the oscillation probabilities.

At neutrino energies above a few hundred $\mathrm{GeV}$ this results in a resonant transition of muon antineutrinos into a sterile state for neutrinos crossing the Earth's core. For neutrinos with energies below $100 \mathrm{GeV}$ the nonzero matter potential and sterile neutrino mixing distort the standard neutrino oscillations pattern. Searches for both effects are performed in IceCube and presented in the following sections.

\section{TeV search for sterile neutrinos}

One of the sterile neutrino searches looks for the resonant transition of muon antineutrinos to the sterile state [13]. The difference between the effective matter potentials for sterile and active neutrinos and the mantle-core-mantle profile of the Earth result in a resonant enhancement of the 

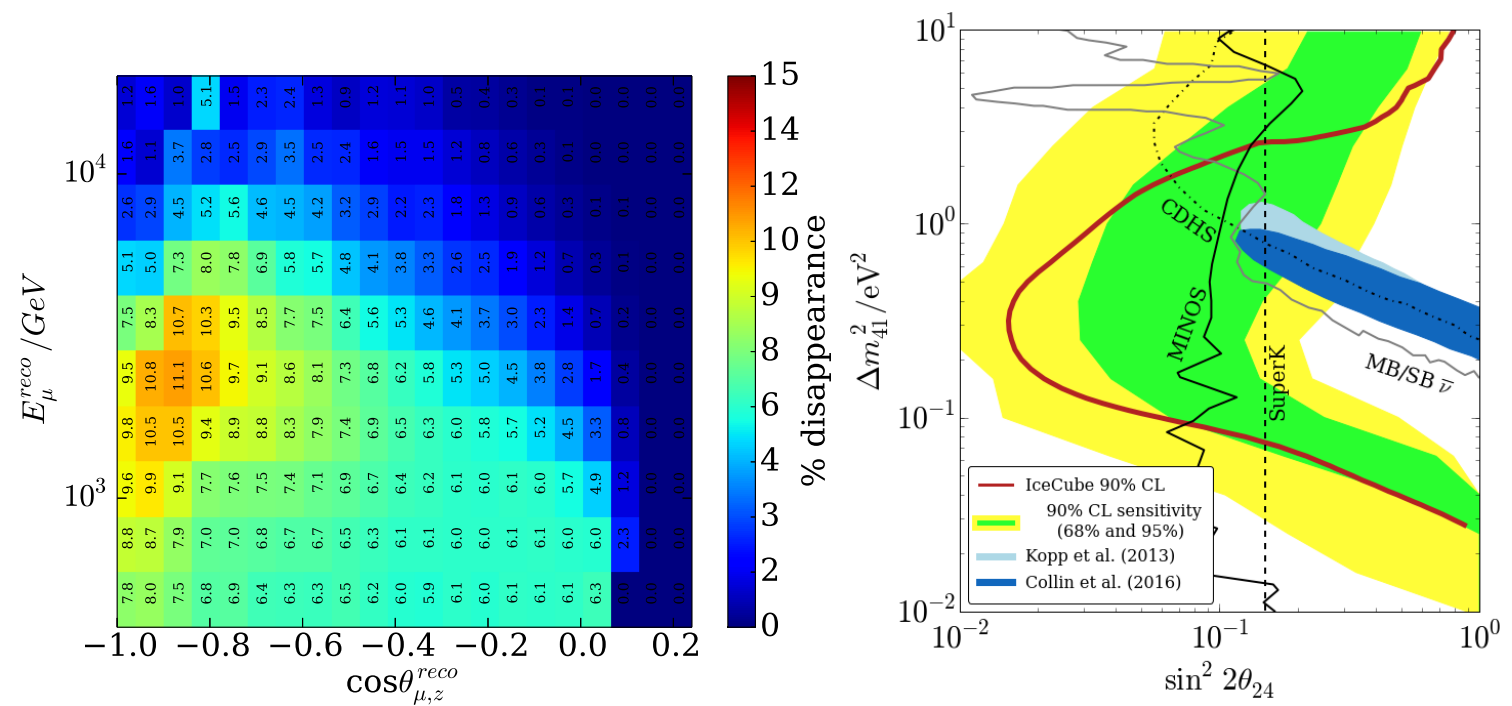

Figure 3: (Left) The expected signature of the sterile neutrino mixing for the final level of the high energy sterile neutrino search with IceCube for the sterile neutrino mixing values $\Delta m_{41}^{2}=1 \mathrm{eV}^{2}$ and $\sin ^{2} 2 \theta_{24}=0.1$. (Right) The $90 \%$ C.L. exclusion limits (red solid line) obtained in the IceCube high energy sterile neutrino search and compared to the limits from previous experiments [18, 19, 20, 21]. Green and yellow shaded areas represent $68 \%$ and $95 \%$ variations of the expected $90 \%$ C.L. sensitivity obtained in simulated pseudoexperiments. The 99\% C.L. allowed regions from global fits in [11] and [22] are shown in light blue and dark blue, respectively.

transition probability $[14,15,16]$. The high energy sterile neutrino search uses the reconstructed neutrino energy range between $400 \mathrm{GeV}$ and $20 \mathrm{TeV}$ to probe the sterile mass splittings between $10^{-2}$ and $10 \mathrm{eV}^{2}$.

An example of the survival probability for muon antineutrinos as they cross the Earth along a diametral (i.e. with zenith angle $\cos \theta_{z}=-1$ ) trajectory is shown in the left part of Figure 2. The density profile of the Earth results in the constructive enhancement of the transition probabilities and a complete disappearance of muon antineutrinos for a specific energy. The energy of the resonant disappearance is proportional to the sterile neutrino mass splitting $\Delta m_{41}^{2}$. The amplitude, zenith angle and the energy of the resonance is sensitive to the mixing angle $\theta_{24}$. The muon antineutrino survival probabilities as function of energy for different sterile neutrino mass splittings are shown in the right part of Figure 2.

The search for the resonant transition is performed using a sample of muons originating from muon neutrino and antineutrino interactions with the antarctic ice. Mainly up-going muons with $\cos \theta_{z}<0.24$ from atmospheric muon neutrinos are used in order to avoid contamination from atmospheric muons. The analysis is performed in the energy range between $400 \mathrm{GeV}$ and $20 \mathrm{TeV}$, where the impact from the standard atmospheric neutrino oscillations is negligible. The data [17] were collected during 343.7 days with the full 86-string configuration of the IceCube detector.

The resulting sample has 20,145 muons with $99.9 \%$ of events being neutrino-induced. The muon zenith angle is reconstructed with a resolution of $\sigma_{\cos \theta_{z}}$ varying between 0.005 and 0.015 . Stochastic losses are used to determine the muon energy with a resolution of $\sigma_{\log _{10} E_{\mu} / \mathrm{GeV}} \sim 0.5$.

The expected impact of the sterile neutrino mixing is shown in the left part of Figure 3. The 

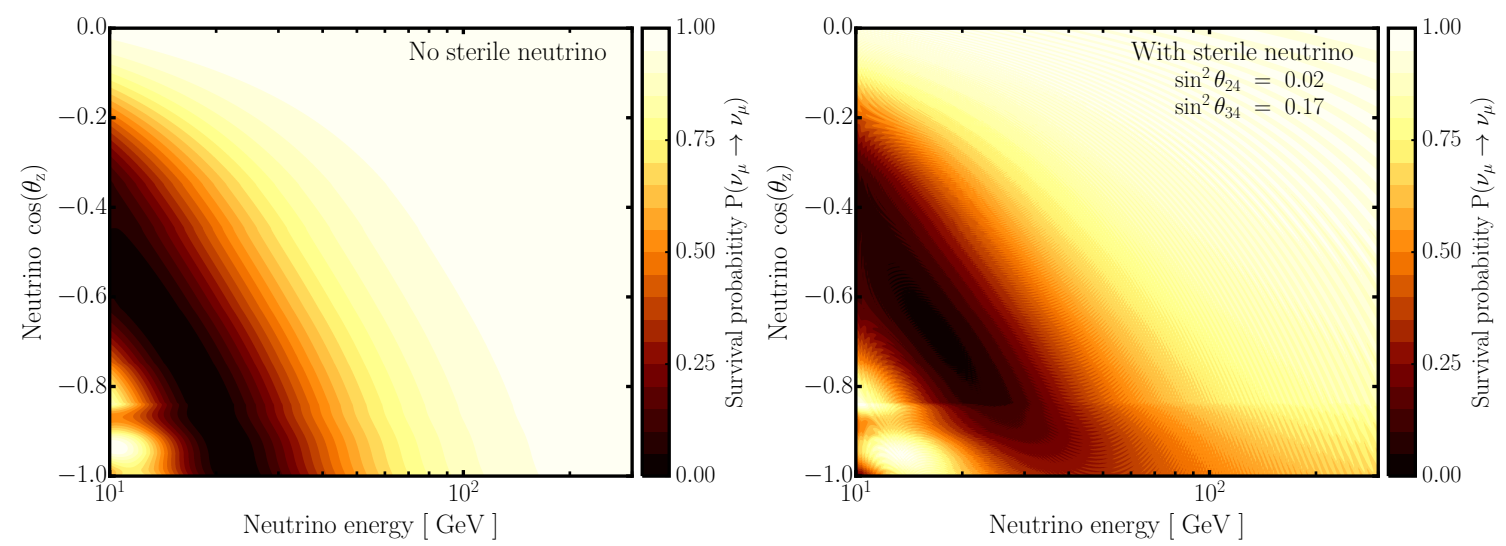

Figure 4: The muon neutrino survival probability as function of energy and neutrino arrival zenith angle for (left) the standard three neutrino model and (right) " $3+1$ " model. The atmospheric neutrino mixing parameters used for the plots are $\Delta m_{32}^{2}=2.51 \cdot 10^{-3} \mathrm{eV}^{2}$ and $\sin ^{2} \theta_{23}=0.51$.

resonant enhancement of the transition probability to the sterile state would result in a distinctive deficit of events in the up-going region. The depth and position of the deficit are used to identify parameters of the mixing between muon and sterile states. The analysis uses a Poisson log-likelihood (LLH) with nuisance parameters to account for systematic uncertainties [13].

The results of the analysis are compatible with the three-neutrino model. The log-likelihood difference to the no-sterile hypothesis of $\triangle L L H=0.75$, corresponding to a p-value of $47 \%$ for the best fit originating from the no-sterile neutrino hypothesis.

This result provides strong constraints on the sterile mixing parameter $\sin ^{2} 2 \theta_{24}$ for mass splittings between 0.1 and $10 \mathrm{eV}^{2}$. The $90 \%$ confidence level (C.L.) allowed region can be limited to

$$
\sin ^{2} 2 \theta_{24}<0.02
$$

at the mass splitting of $\Delta m_{41}^{2} \sim 0.3 \mathrm{eV}^{2}$. The resulting $90 \%$ C.L. exclusion limit as function of the mass splitting is shown in the right part of Figure 3. This study excludes the global estimates of the mixing from the short baseline experiments [11, 23], including LSND and MiniBooNE, at approximately $99 \%$ C.L., excluding a large fraction of the allowed parameter space for the " $3+1$ " model. Updated global fits, such as [24], demonstrate the impact of this result on a globally allowed region of the sterile neutrino mixing.

\section{Low energy search}

Another sterile neutrino search [25] is performed in the neutrino energy range between 6 and $56 \mathrm{GeV}$. It searches for the deviations from the standard oscillations pattern caused by sterile neutrino mixing. Above $10 \mathrm{GeV}$, the muon survival probability can be approximated ${ }^{1}$ as

$$
P\left(v_{\mu} \rightarrow v_{\mu}\right) \approx 1-\sin ^{2}\left(2 \theta_{23}\right) \sin ^{2}\left(\Delta m_{32}^{2} \frac{L}{4 E_{v}}\right),
$$

\footnotetext{
${ }^{1}$ The probability in the two-neutrino approximation is shown for simplicity here, however the full three or $3+1$ flavor probability is used in the analysis and results.
} 
where $\Delta m_{32}^{2}$ is the atmospheric neutrino mass splitting, $\theta_{23}$ is the corresponding mixing angle, $L$ is the distance traveled from the production point and $E_{v}$ is the neutrino energy. The values of $\Delta m_{32}^{2}$ and $\theta_{23}$ define the period and depth of the neutrino oscillations, respectively. The values of the mixing parameters [1] lead to an almost complete disappearance of muon neutrinos with energies of $25 \mathrm{GeV}$ crossing the Earth along its diameter. The survival probability for atmospheric muon neutrinos as a function of neutrino energy and arrival zenith angle are shown in the left part of Figure 4.

In the case of non-zero sterile neutrino mixing the atmospheric neutrino oscillations no longer follow equation (5.1) $[12,26,16]$. The frequency of the oscillations as well as their amplitude are changed for non-zero values of $U_{\alpha 4}$ elements of the mixing matrix in Equation (3.1). An example of such a change is shown in the right part of Figure 4. The effect is proportional to the amount of matter along the neutrino trajectory and is most pronounced for neutrinos crossing the Earth's core and minimal for neutrinos arriving from the horizon. The effect of the sterile neutrino mixing in the energy range of $5-56 \mathrm{GeV}$ is independent from the value of the sterile mass splitting, because fast oscillations are averaged by the detector resolutions for $\Delta m_{41}^{2} \geq 0.3 \mathrm{eV}^{2}$. The value of $\Delta m_{41}^{2}$ is fixed to $1 \mathrm{eV}^{2}$ throughout the analysis.

This study uses three years (2011-2013) of IceCube DeepCore data [27], comprising 5118 events in total. Only well reconstructed up-going neutrino events in the energy range between 6 and $56 \mathrm{GeV}$ are used. The event selection originates from the measurement of the atmospheric mixing parameters [28]. It is designed to suppress the background from atmospheric muons and identify muon tracks produced by charged current interactions of atmospheric muon neutrinos. Minor improvements in the muon rejection and reconstruction techniques were performed in this study [25].

The light detected outside the DeepCore fiducial volume is used to reject atmospheric muon background. An additional veto region is added below the dust layer (see Figure 1) to reduce contamination from atmospheric muons penetrating to DeepCore through the dust layer and, therefore, not triggering DOMs in the outer layers of IceCube. Special algorithms are designed to search for muons penetrating to DeepCore through non-instrumented corridors formed by the detector geometry. An example of such a corridor is shown in the left part of Figure 1. A data driven template is used to estimate the atmospheric muons at the final level of the analysis. The data sample is estimated to have about $0.4 \%$ contamination due to remaining atmospheric muons.

A directional reconstruction uses a specific light pattern of non-scattered photons [29, 30]. The median muon neutrino zenith resolution is $10^{\circ}$ at $10 \mathrm{GeV}$ and improves to $6^{\circ}$ at $40 \mathrm{GeV}$. Deep Inelastic Scattering (DIS) is the leading neutrino interaction process for the energies considered in this study. A muon track and hadronic shower are typically produced in DIS interactions of muon neutrinos. The total energy is estimated as a sum of hadronic shower and muon. The neutrino energy resolution is about $30 \%$ at $8 \mathrm{GeV}$ and improves to $20 \%$ at $20 \mathrm{GeV}$.

The expected impact of a sterile neutrino mixing on the final level of the analysis is shown in the left part of Figure 5. A Poisson log-likelihood with nuisance parameters is maximized to find the best estimate for the sterile neutrino mixing parameters. Twelve nuisance parameters are used to account for the systematic uncertainties from the standard atmospheric mixing parameters, neutrino fluxes, detector uncertainties, cross sections and background. Confidence levels are estimated using Wilks' theorem [31]. Both standard neutrino mass orderings were considered in the analysis. 

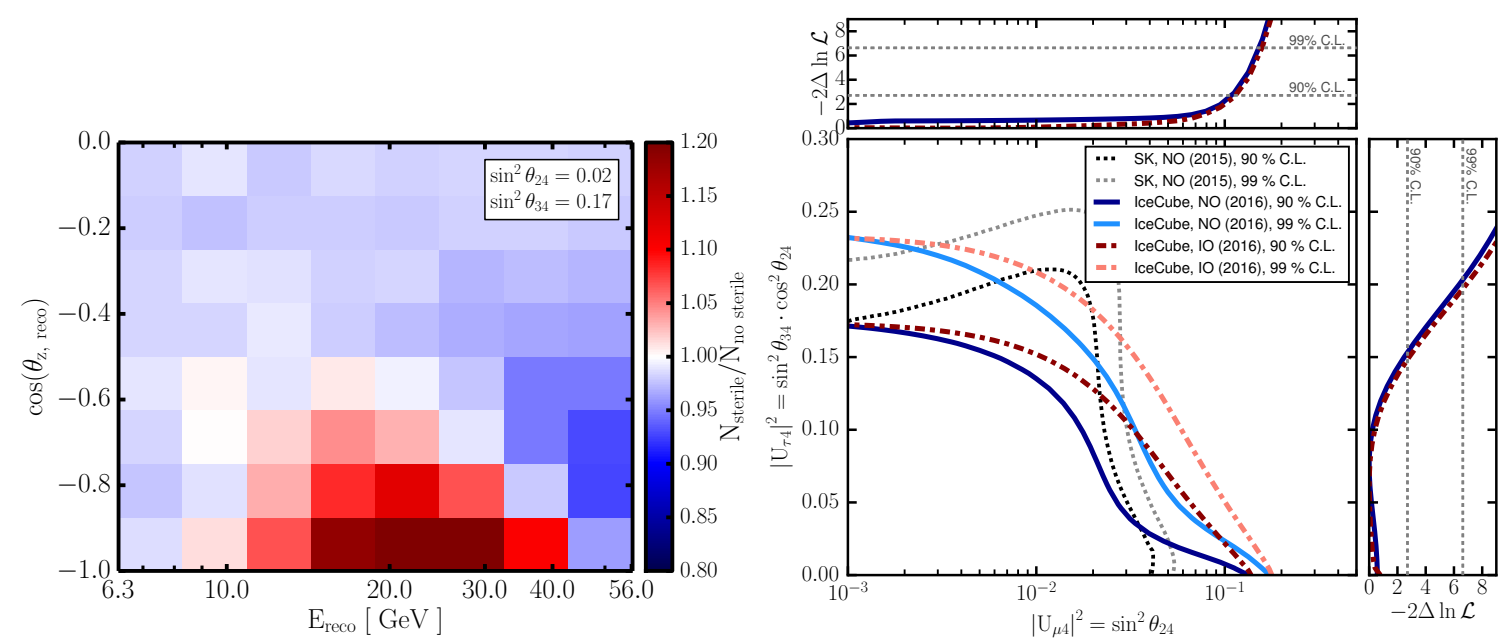

Figure 5: (Left) The expected signature of sterile neutrino mixing in the IceCube DeepCore search. The effect of the sterile neutrino mixing is independent of the value of the sterile mass splitting for $\Delta m_{41}^{2} \leq 0.3$ $\mathrm{eV}^{2}$. (Right) The exclusion limits on the sterile neutrino mixing obtained in the IceCube DeepCore search for normal (NO, $m_{4}>m_{3}>m_{2}>m_{1}$ ) and inverted (IO, $m_{4}>m_{2}>m_{1}>m_{3}$ ) neutrino mass orderings. The black curve represents the limits obtained by the Super-Kamiokande experiment [18].

The results of the analysis are found to be consistent with the standard three neutrino paradigm, with a $\log$-likelihood difference $-2 \Delta \ln \mathscr{L}=0.8$, corresponding to a p-value of about $30 \%$ for the best fit to originate from statistical fluctuations of the no sterile neutrino hypothesis. The estimation is derived from 2000 statistical trials.

The exclusion limits are obtained by scanning the log-likelihood space for the mixing elements $\left|U_{\mu 4}\right|^{2}$ and $\left|U_{\tau 4}\right|^{2}$ and are presented in the right part of Figure 5. The corresponding limits on the elements of the mixing matrix are

$$
\begin{aligned}
\left|U_{\mu 4}\right|^{2} & <0.11(90 \% \text { C.L. }), \\
\left|U_{\tau 4}\right|^{2} & <0.15 \text { (90\% C.L.). }
\end{aligned}
$$

Currently, the results of this search set the leading constraints on the $\left|U_{\tau 4}\right|^{2}$ element of the mixing matrix. The sensitivity of this study to $\left|U_{\tau 4}\right|^{2}$ is limited by a number of factors, including atmospheric neutrino flux uncertainties and the detector resolutions. The best constraints on $\left|U_{\mu 4}\right|^{2}$ are coming from the IceCube study described in the previous section and from the MINOS experiment [19].

\section{Discussion and outlook}

Two approaches to search for sterile neutrino mixing using the data from IceCube are presented in this work. They provide strict constraints on the allowed sterile neutrino mixing with muon and tau neutrinos in a " $3+1$ " model.

Currently six years of the full 86-string detector configuration have been recorded. The high energy sterile neutrino search uses only 1 year of this data. More years of data recorded by IceCube 
as well as improvements in event selections and reconstructions are expected to provide better sensitivity to the sterile neutrino mixing.

The low energy search uses only three years of data. Monte Carlo studies show that the current limits are driven by statistical uncertainties. Therefore, the sensitivity can be improved by using the full data available in IceCube. Extending the energy range will add more information about the neutrino flux and may reduce the impact of systematic uncertainties. Inclusion of $v_{e}$ and $v_{\tau}$ components of the atmospheric neutrino flux, as well as neutral current interactions can improve the sensitivity even further.

\section{References}

[1] I. Esteban, M. C. Gonzalez-Garcia, M. Maltoni, I. Martinez-Soler and T. Schwetz, Updated fit to three neutrino mixing: exploring the accelerator-reactor complementarity, JHEP 2017 (Jan, 2017) 87, [arXiv:1611.01514].

[2] LSND COllaboration collaboration, A. Aguilar et al., Evidence for neutrino oscillations from the observation of $\bar{v}_{e}$ appearance in a $\bar{v}_{\mu}$ beam, Phys. Rev. D64 (Nov, 2001) 112007.

[3] MiniBoone Collaboration collaboration, A. A. Aguilar-Arevalo et al., Improved Search for $\bar{v}_{\mu} \rightarrow \bar{v}_{e}$ Oscillations in the MiniBooNE Experiment, Phys. Rev. Lett. 110 (Apr, 2013) 161801.

[4] G. Mention, M. Fechner, T. Lasserre, T. A. Mueller, D. Lhuillier, M. Cribier et al., Reactor antineutrino anomaly, Phys. Rev. D83 (Apr, 2011) 073006.

[5] J. N. Abdurashitov et al., Measurement of the response of a Ga solar neutrino experiment to neutrinos from a ${ }^{37}$ Ar source, Phys. Rev. C 73 (Apr, 2006) 045805.

[6] Aleph Collaboration, Delphi Collaboration, L3 Collaboration, OPAL Collaboration, SLD Collaboration, LEP Electroweak Working Group, SLD Electroweak Group, SLD Heavy Flavour Group collaboration, S. Schael et al., Precision electroweak measurements on the Z resonance, Phys. Rep. 427 (2006) 257 - 454, [arXiv:hep-ex/0509008].

[7] IceCube Collaboration collaboration, M. Aartsen et al., The IceCube Neutrino Observatory: Instrumentation and Online Systems, JINST 12 (2017) P03012, [arXiv: 1612 . 05093].

[8] ICECuBe Collaboration collaboration, R. Abbasi et al., The design and performance of IceCube DeepCore, Astropart. Phys. 35 (2012) 615 - 624, [arXiv:1109.6096].

[9] S. Bilenky and B. Pontecorvo, Lepton mixing and neutrino oscillations, Phys. Rep. 41 (1978) 225 261.

[10] Z. Maki, M. Nakagawa and S. Sakata, Remarks on the Unified Model of Elementary Particles, Prog. Theor. Phys. 28 (1962) 870-880.

[11] J. Kopp, P. A. N. Machado, M. Maltoni and T. Schwetz, Sterile neutrino oscillations: the global picture, JHEP 05 (2013) 050, [arXiv: 1303.3011$].$

[12] S. Razzaque and A. Y. Smirnov, Searching for sterile neutrinos in ice, JHEP 2011 (2011) 84, [arXiv:1104.1390].

[13] IceCube Collaboration collaboration, M. G. Aartsen et al., Searches for Sterile Neutrinos with the IceCube Detector, Phys. Rev. Lett. 117 (Aug, 2016) 071801. 
[14] Q. Liu and A. Smirnov, Neutrino mass spectrum with $v_{\mu} \rightarrow v_{s}$ oscillations of atmospheric neutrinos, Nuclear Physics B 524 (1998) 505 - 523, [arXiv: hep-ph/9712493].

[15] M. V. Chizhov and S. T. Petcov, Enhancing mechanisms of neutrino transitions in a medium of nonperiodic constant-density layers and in the Earth, Phys. Rev. D 63 (Mar, 2001) 073003, [arXiv: hep-ph/9903424].

[16] H. Nunokawa, O. Peres and R. Zukanovich Funchal, Probing the \{LSND\} mass scale and four neutrino scenarios with a neutrino telescope, Phys. Lett. B 562 (2003) 279 - 290, [arXiv:hep-ph/0302039].

[17] http://icecube.wisc.edu/science/data/IC86-sterile-neutrino/.

[18] Super-Kamiokande Collaboration collaboration, K. Abe et al., Limits on sterile neutrino mixing using atmospheric neutrinos in Super-Kamiokande, Phys. Rev. D91 (Mar, 2015) 052019.

[19] MiNOS Collaboration collaboration, P. Adamson et al., Search for Sterile Neutrinos Mixing with Muon Neutrinos in MINOS, Phys. Rev. Lett. 117 (Oct, 2016) 151803, [1607.01176].

[20] MiniBoone and SciBoone Collaborations collaboration, G. Cheng et al., Dual baseline search for muon antineutrino disappearance at $0.1 \mathrm{ev}^{2}<\Delta m^{2}<100 \mathrm{ev}^{2}$, Phys. Rev. D 86 (Sep, 2012) 052009, [1208.0322].

[21] F. Dydak et al., A search for $v_{\mu}$ oscillations in the $\Delta m^{2}$ range $0.3-90 \mathrm{eV}^{2}$, Physics Letters $B 134$ (1984) $281-286$

[22] G. H. Collin, C. A. Argüelles, J. M. Conrad and M. H. Shaevitz, Sterile Neutrino Fits to Short Baseline Data, Nucl. Phys. B908 (2016) 354-365, [1602.00671].

[23] J. M. Conrad, C. M. Ignarra, G. Karagiorgi, M. H. Shaevitz and J. Spitz, Sterile Neutrino Fits to Short Baseline Neutrino Oscillation Measurements, Adv. High Energy Phys. 2013 (2013) 163897, [arXiv:1207.4765].

[24] S. Gariazzo, C. Giunti, M. Laveder and Y. F. Li, Updated global 3+1 analysis of short-baseline neutrino oscillations, Journal of High Energy Physics 2017 (Jun, 2017) 135, [1703. 00860 ].

[25] IceCube Collaboration collaboration, M. G. Aartsen et al., Search for sterile neutrino mixing using three years of IceCube DeepCore data, Phys. Rev. D 95 (Jun, 2017) 112002, [1 702.05160 ].

[26] S. Razzaque and A. Y. Smirnov, Searches for sterile neutrinos with IceCube DeepCore, Phys. Rev. D 85 (May, 2012) 093010, [arXiv:1203.5406].

[27] http://icecube.wisc.edu/science/data/nu_osc/.

[28] IceCube Collaboration collaboration, M. G. Aartsen et al., Determining neutrino oscillation parameters from atmospheric muon neutrino disappearance with three years of IceCube DeepCore data, Phys. Rev. D91 (Apr, 2015) 072004.

[29] J. Aguilar et al., A fast algorithm for muon track reconstruction and its application to the \{ANTARES\} neutrino telescope, Astropart. Phys. 34 (2011) 652 - 662, [1105.4116].

[30] J. P. Yáñez Garza, Measurement of neutrino oscillations in atmospheric neutrinos with the IceCube DeepCore detector, Ph.D. thesis, Humboldt-Universität zu Berlin, 2014.

[31] S. S. Wilks, The large-sample distribution of the likelihood ratio for testing composite hypotheses, Ann. Math. Statist. 9 (03, 1938) 60-62. 